\title{
Метод компенсации нелинейных искажений сигнала в WDM системах связи на основе теории возмущений и машинного обучения
}

\author{
Е.А. Аверьянов ${ }^{1, *}$, А.А. Редюк ${ }^{1,2}$, О.С. Сидельников ${ }^{1,2}$, \\ М.А. Сорокина ${ }^{3}$, М.П. Федорук ${ }^{1,2}$, С.К. Турицын ${ }^{1,3}$ \\ ${ }^{1}$ Новосибирский государственный университет, Новосибирск \\ ${ }^{2}$ Институт вычислительных технологий СО РАН, Новосибирск \\ ${ }^{3}$ Институт фотонных технологий, Университет Астона, Бирмингем, Великобритания \\ *E-mail: euav@ya.ru
}

DOI:10.31868/RFL2018.239-241

Нелинейные искажения, возникающие в волоконно-оптических линиях связи (ВОЛС), остаются одним из основных ограничивающих факторов, препятствующих дальнейшему увеличению дальности и скорости передачи информации. В особенности это касается WDM систем, подверженных, помимо внутриканальных, также и межканальным нелинейным взаимодействиям. Для компенсации данного эффекта было предложено множество способов [1-4]. Однако, практическая реализация этих методов сопряжена с рядом трудностей.

В частности, в статье [5] авторы рассматривают модель межканального нелинейного взаимодействия на примере двух каналов с двумя поляризациями. Для её применения необходимо вычислять тысячи модельных коэффициентов возмущений, выраженных многомерными осциллирующими интегралами, число которых только растёт с увеличением числа каналов. Вычислительная сложность потенциально может быть уменьшена с помощью привлечения обширного арсенала методов машинного обучения, бурно развивающихся в области ВОЛС последние несколько лет [6]. Как предлагается авторами [7], вместо численного интегрирования возможно применение робастной регрессионной модели. Такой подход демонстрирует высокую эффективность при значительно уменьшенной трудоёмкости для систем с пространственным уплотнением каналов.

В данной работе мы обобщаем этот метод для WDM систем для компенсации внутриканальных и межканальных нелинейных эффектов, используя информацию с соседних по частоте каналов, чтобы восстановить изначальный сигнал.

Анализ возмущений первого порядка уравнений Манакова позволяет получить приближенные оценки накопленных во время распространения в волоконно-оптической среде нелинейных искажений сигнала в аддитивной форме $X_{k}^{s}=x_{k}^{s}+\Delta x_{k}^{s}$, где $x_{k}^{s}$ и $X_{k}^{s}$ комплексные амплитуды сигнала во временном слоте $k$ в -поляризации в канале $s$ на передатчике и приёмнике соответственно. Тогда само нелинейное искажение записывается следующим образом:

$$
\begin{gathered}
\Delta x_{k}^{S}=\sum_{m, n} C_{m n}\left(x_{k+m}^{s} x_{k+n}^{s} \bar{x}_{k+m+n}^{s}+x_{k+m}^{S} y_{k+n}^{S} \bar{y}_{k+m+n}^{s}\right)+ \\
\sum_{c \neq s} \sum_{m, n} D_{m n}^{c}\left(2 x_{k+m}^{s} x_{k+n}^{c} \bar{x}_{k+m+n}^{c}+x_{k+m}^{s} y_{k+n}^{c} \bar{y}_{k+m+n}^{c}+y_{k+m}^{s} x_{k+n}^{c} \bar{y}_{k+m+n}^{c}\right),
\end{gathered}
$$

где $C_{m n}$ и $D_{m n}^{c}$ - модельные коэффициенты возмущений. Заменой символов $x$ на $y$ и наоборот получаются аналогичные выражения для $y$-поляризации.

Основная идея предлагаемого метода состоит в поиске коэффициентов возмущений используя методы машинного обучения на заранее известном тренировочном наборе отправленных и принятых символов. После обучения, найденные коэффициенты применяются для компенсации по формуле $x_{k}^{s}=X_{k}^{s}-$ 
$\Delta x_{k}^{S}$, где для оценки $\Delta x_{k}^{S}$ и $\Delta y_{k}^{S}$ используется принятый сигнал после обработки линейным эквалайзером в качестве приближения отправленных символов.

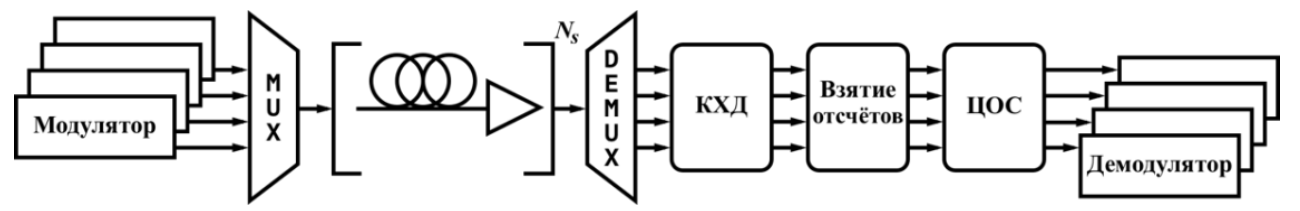

Рис. 1. Принципиальная схема исследуемой ВОЛС.

Чтобы оценить эффективность предлагаемого метода посткомпенсации, основанного на возмущениях первого порядка (ВПК), был проведён численный эксперимент передачи данных по ВОЛС, принципиальная схема которой представлена на Рис. 1. В рассмотрении находилась трёхканальная передача RRC импульсов с форматом модуляции 16QAM и символьной скоростью 32 Гбод на длине несущей волны 1550 нм, межканальное расстояние 37,5 ГГц. В линии используются точечные усилители после каждого из 10 пролётов длиной 100 км с коэффициентом шума 4,5 дБ. На приёмнике после демультиплексирования применяется идеальная компенсация хроматической дисперсии, взятие отсчётов с согласованным фильтром и цифровая обработка сигнала.

Распространение сигнала в модели описывается уравнением Манакова

$$
\frac{\partial A_{x, y}}{\partial z}=\left[-\frac{\alpha}{2}-i \frac{\beta_{2}}{2}+i \gamma \frac{8}{9}\left(\left|A_{x}\right|^{2}+\left|A_{y}\right|^{2}\right)\right] A_{x, y},
$$

где $A_{x, y}$ комплексная огибающая электрического поля для $x$ - и -поляризаций, коэффициент затухания $\alpha=0,2$ дБ/км, коэффициент дисперсии второго порядка

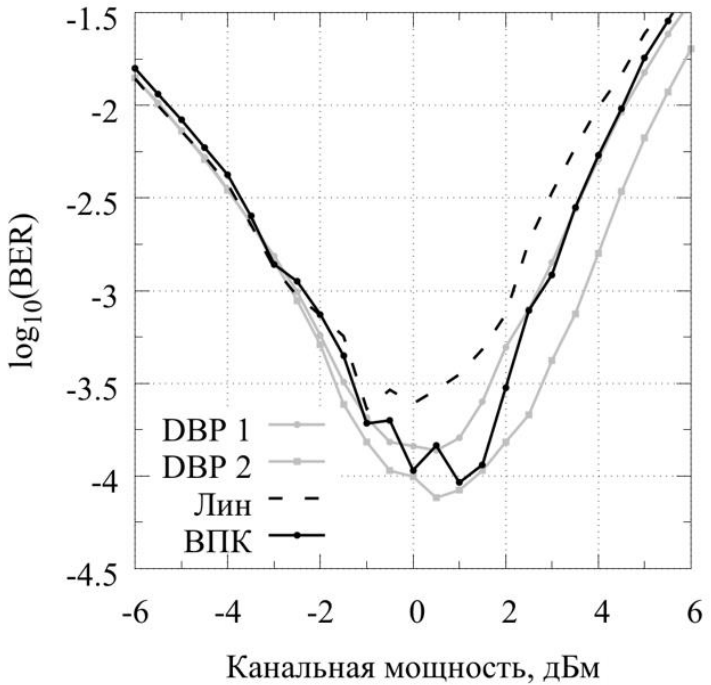

Рис. 2. Сравнение эффективности методов. $\beta_{2}=17$ пс/нм/км, коэффициент нелинейности $\gamma=1,4$ 1/Вт/км. Данные уравнения решались численно с помощью симметричной схемы метода Фурье расщепления по физическим процессам с 16 отсчётами на символ.

На Рис. 2 представлена эффективность ВПК в терминах BER в сравнении с линейным эквалайзером (Лин) и методом обратного распространения для центрального канала с одним и двумя шагами на пролёт $\quad$ (DBP 1 и соответственно). Как видно, ВПК работает на уровне промежуточном между DBP 1 и $\mathrm{DBP} 2$ для оптимальной мощности. При это для ВПК требуется значительно меньше вычислительных операций. Такой результат хорошо согласуется со случаем трёх пространственных каналов в смежной работе [7].

Исследование выполнено при финансовой поддержке Российского фонда фундаментальных исследований (грант № 17-42-543379).

\section{Литература}

[1] E. Ip, J. Lightwave Technology 28, 939-951 (2010)

[2] L. Liu et al, J. Lightwave Technology 30, 310-316 (2012) 
[3] T. Oyama et al, Proc. OFC, Tu3A.3, San Francisco (2014)

[4] Z. Tao et al, J. Lightwave Technology 29, 2570-2576 (2011)

[5] R. Dar et al, J. Lightwave Technology 33, 1044-1053 (2015)

[6] D. Zibar et al, J. Lightwave Technology 34, 1442-1452 (2016)

[7] M. Sorokina et al, Opt. Express 24, pp. 30433-30443 (2016) 